\title{
Editorial
}

\section{Controversies in the Management of Soft Tissue Injuries}

\author{
Wasim S. Khan ${ }^{*}, 1$, and Atif Malik ${ }^{2}$
}

\author{
${ }^{I}$ University College London Institute of Orthopaedics and Musculoskeletal Sciences, Royal National Orthopaedic \\ Hospital, Stanmore, Middlesex, HA7 4LP, UK \\ ${ }^{2}$ Department of Trauma and Orthopaedics, Queens Hospital, Barking Havering and Redbridge NHS Trust, Romford, \\ Essex, RM7 0AG, UK
}

Soft tissue injuries include a wide and heterogeneous range of injuries. In this issue we cover a range of these topics including negative pressure wound therapy and the evidence-based principles behind its use, multiligamentous knee injuries, knee dislocations, compartment syndrome, and nerve injuries and principles of repair. There are a number of papers on bite injuries ranging from a literature review, their microbiology and virology, and mammalian bites. We also include a research paper on the management of knee osteochondritis dissecans. No issue on soft tissues in an orthopaedic journal would be completed without a review on fractures, and that too is included.

The use of Negative Pressure Wound Therapy (NPWT) for complex and large wounds has increased in popularity over the past decade. Modern NPWT systems consisting of an open pore foam sponge, adhesive dressing and a vacuum pump producing negative pressure have been used as an adjunct to surgical debridement to treat tissue defects around open fractures and chronic, contaminated wounds. Other uses include supporting skin grafts and protecting wounds at risk of breaking down. A review by Putnis et al. outlines the current and emerging indications for negative pressure wound therapy in Orthopaedic trauma and the existing preclinical and clinical evidence base for its use. A review by Novak et al. explores the available evidence and describes future developments for the use of NPWT in trauma and orthopaedic practice.

Multiligamentous knee injuries are rare but serious injuries can threaten limb viability. As such, they require careful management to give patients the best chance of immediate and ultimate functional recovery. However, as these injuries are rare, there is paucity in prospective comparative studies large enough to provide high level evidence for best practice. This lack of comprehensive and convincing evidence has made the management of multiligamentous knee injuries an area of active debate and controversy. The debate on whether surgical management

*Address correspondence to this author at the University College London Institute of Orthopaedics and Musculoskeletal Sciences, Royal National Orthopaedic Hospital, Stanmore, Middlesex, HA7 4LP, UK;

Tel: 07791 025554; Fax: 01707 655059;

E-mail: wasimkhan@doctors.org.uk

${ }^{\S}$ Guest Editor. leads to better outcomes than non-operative management, the optimal timing of surgery after injury and whether repair is better than reconstruction is still ongoing. Using the Oxford Levels of Evidence, a review by Mabvuure et al. summarises the latest high level evidence to provide answers to these issues. Recommendations for practice have also been offered and assigned a grade using a published scale.

Traumatic knee dislocation is a serious and potentially limb threatening injury that can be easily missed if meticulous history and examination have not been employed. Neurovascular injuries are common in this condition, and due diligence should be given to their thorough evaluation at time of secondary survey so as to avoid complications such as ischaemia, compartment syndrome and eventual amputation. There is growing evidence in the literature that morbid obesity is associated with low energy knee dislocation, therefore this should be considered when assessing this cohort of patients presenting with an acute knee injury. Early operative intervention especially with multiligaments involvement is the preferable strategy in the management of this acute injury. Controversy exists whether to reconstruct or repair damaged structures, and whether to adopt a one stage or two stage reconstruction of the cruciate ligaments. Early rehabilitation is important and essential to achieve satisfactory outcomes. The article by McKee et al. is an evidence-based overview of this rare but devastating injury.

Acute compartment syndrome (ACS) is a surgical emergency warranting prompt evaluation and treatment. It can occur with any elevation in interstitial pressure in a closed osseo-fascial compartment. Resultant ischaemic damage may be irreversible within six hours and can result in long-term morbidity and even death. The diagnosis is largely clinical with pain out of proportion to the injury being the commonest symptom. Compartment pressure monitors can be a helpful adjunct where the diagnosis is in doubt. Initial treatment is with the removal of any constricting dressings or casts, avoiding hypotension and maintaining the limb at the level of the heart. If symptoms persist definitive treatment is with timely surgical decompression of all the involved compartments. The article by Donaldson et al. reviews the pathophysiology, diagnosis and current management of ACS. 
Peripheral Nerve Injuries are one of the most common causes of hand dysfunction caused by upper limb trauma but still current management has remained suboptimal. The review by Griffin et al. aims to explain the traditional view of pathophysiology of nerve repair and also describe why surgical management is still inadequate using the new biological research that has documented the changes that occur after the nerve injury, which, could cause suboptimal clinical outcomes. Subsequently, presentation and diagnosis will be described for peripheral nerve injuries. When traditional surgical repair using end-to-end anastomosis is not adequate, nerve conduits are required with the gold standard being the autologous nerve. Due to associated donor site morbidity and poor functional outcome documented with autologous nerve repair several new advancements for alternatives to bridge the gap are being investigated. We will summarise the new and future advancements of non-biological and biological replacements as well as gene therapy, which are being considered as the alternatives for peripheral nerve repair.

Patients presenting to the emergency department with bite injuries to the hand sustain them through a number of causes including domesticated as well as stray animal bites, and human bites commonly sustained as a result of violence. The nature of the injuries sustained can be very deceptive. A small tooth mark on the exterior can be a fulminant infection in the tissues deep down. Tendon injuries, fractures of the metacarpals and phalanges and management of the wound are critical issues faced by a surgeon in dealing with such patients. Similarly the less common bite injuries to the hand, often with disastrous and sometimes fatal complications, do also present to the emergency department. A high incidence of suspicion is needed in dealing with these injuries effectively. In their article, Raval et al. discuss the common as well as uncommon causes of bite injuries to the hand and their management. In addition to reviewing the literature to ascertain the management of such injuries, they also discuss interesting and rare case reports. Malahias et al. discuss the associated microbiology and virology of these injuries as well as their management. Jha et al. review the literature and discuss the epidemiology, pathophysiology and microbiology relating specifically to mammalian bite injuries, as well as clinical aspects including signs, symptoms, and management.

Surgical fixation is recommended for stable osteochondritis dissecans (OCD) lesions that have failed non-operative management and for all unstable lesions. In their study, Pengas et al. set out to describe and evaluate an alternative method of surgical fixation for such lesions. Five knees with unstable OCD lesions in four male adolescent patients with open physes were treated with the AO Hook Fixation System. The outcome was evaluated both clinically and with three separate outcome systems (IKDC 2000, KOOS, Lysholm) at one and a mean four year follow-up. We demonstrated excellent clinical results in all patients. At four years, all scoring systems demonstrated statistically significant improvement when compared to the preoperative status. Their study suggests that the AO Hook Fixation System is an alternative method of surgical intervention with comparable medium term results with other existing modes of fixation and the added biomechanical advantage of the absence of distracting forces during hardware removal.

Jordan \& Modi discuss current concepts in the management of proximal humeral fractures. Proximal humeral fractures are common and although the majority can be managed non-operatively, the optimal treatment of displaced or complex fractures remains controversial. Nonoperative treatment is typically selected for minimally displaced fractures where union rates are high and good or excellent outcomes can be expected in approximately $80 \%$ of cases. The aims of surgical fixation are to restore articular surface congruency, alignment and the relationship between the tuberosities and the humeral head. Hemiarthroplasty provides patients with reliable pain relief and its indications include fracture dislocations, humeral head splitting fractures and some three- and four-part fractures. The key areas of surgical technique that influence functional outcome include correctly restoring the humeral height, humeral version and tuberosity position. Function, however, is poor if the tuberosities either fail to unite or mal-unite. The interest in reverse shoulder arthroplasty as an alternative option has therefore recently increased, particularly in older patients with poor bone quality and tuberosity comminution. The evidence supporting this, however, is currently limited to multiple case series with higher level studies currently underway.

O'Brien et al. discuss controversies in the management of open fractures. Open fractures are a common problem encountered by orthopaedic surgeons and comprise a broad spectrum of trauma. Management is guided by principlebased steps aimed at reducing the risk of gas gangrene or suppurative infections, whilst maintaining viability in a favourable soft tissue environment to reduce the risk of delayed or non-union of bone. Aspects of these principles, however, create discussion around several areas of controversy. The specific antimicrobial regimen and its duration are questions that have been evaluated for decades. Like the ever-evolving nature of the bacterial pathogens, the answer to this is also dynamic and changing. The "six-hour rule" is also a hotly debated topic with fervent perseverance of this dogma despite a gross lack of support from the literature. The most appropriate soft tissue management approach for open fractures - immediate definitive soft tissue closure versus leaving wounds open for delayed closure or definitive management - is also an area of debate. Exploration of these controversies and consideration for the historical context of the supporting literature furthers our understanding of the critical elements.

We hope that all physicians, surgeons, therapists and practitioners involved in the care of soft tissue injuries find this issue useful.

(C) Khan and Malik; Licensee Bentham Open.

This is an open access article licensed under the terms of the Creative Commons Attribution Non-Commercial License (http://creativecommons.org/licenses/by-nc/3.0/) which permits unrestricted, non-commercial use, distribution and reproduction in any medium, provided the work is properly cited. 